\title{
LARGE MULTIBEAM ARRAY ANTENNAS WITH REDUCED NUMBER OF ACTIVE CHAINS
}

\author{
G. Caille (*), Y. Cailloce (*), C. Guiraud (*) ; D. Auroux (**), T. Touya (**), M.Masmousdi (**) \\ (*) Thales Alenia Space, BP 33787, 31037 Toulouse, France - gerard.caille@thalesaleniaspace.com \\ (**) Applied Mathematics Laboratory (MIP), Paul Sabatier University (UPS), Toulouse, France - auroux@mip.ups-tlse.fr
}

Keywords: Array antenna, multi-beam, multimedia via satellite

\begin{abstract}
Direct Radiating Arrays ('DRA') have been proven to be an interesting solution for reconfigurable multibeam transmit antennas, as spreading naturally the RF power to be radiated over the whole aperture, and avoiding cold redundancies thanks to graceful degradation. DRA are in general designed following two main constraints :

- Antenna diameter is determined by directivity and isolation specifications

- Grid lattice is constrained by grating lobe rejection outside a given domain (typically outside the Earth, for geostationary satellite antennas considered here)
\end{abstract}

As high directivity beams are mostly required, adding these two constraints leads to a prohibitive number of antenna elements (so of active chains).

In order to reduce the number of active chains without affecting antenna pattern characteristics, two solutions are studied here :

- Array thinning, relies on suppressing part of the radiating elements in the regular grid lattice,

- $\quad$ Non regular aperture sampling consists in dividing the radiating aperture into non-regular subarrays. Industrial constraints lead to gather small identical elements in rectangular groups with various size. The basic elements are small enough to avoid any grating lobe on the Earth disk; as the $2^{\text {nd }}$-step aperture meshing (by non-regular groups) is nonperiodic, no other grating lobes appear on the Earth.

Various kinds of mathematical algorithms have been compared for both these arraying methods. They are classified in 2 main categories:

a) part I presents so-called "global optimisation algorithms based on random searching": 'genetic' algorithm and 'simulated annealing' are assessed to perform array thinning and subarray division. As a result, such algorithms are wellsuited to array thinning, but not to gathering elements in nonregular groups, providing good performances for all numerous beams; so we went to a new category of much different methods

b) part II presents a new analytical method, built especially for this problem by UPS/MIP. It associates:

- $\quad$ an optimised choice of the cost function, able to warrant convergence of a gradient-type method

- combining solutions found for each beam in a single power distribution on the aperture is performed using the "Singular Value Decomposition" (SVD) method

- then the obtained distribution is sampled into amplitude values that can be provided by gathering elements by $1,2,3$, or 4 . And the best rectangles arrangement is found in an iterative process using "topologic gradient" method.

A clever association of these various steps leads to a nonregular subarrays distribution saving nearly $50 \%$ of the initial elements number, while complying for all beams with typical requirements on gain and isolation, and using equal-power feeding, so better efficiency and lower cost for a single amplifiers class.

\section{Introduction: need for reducing the number of active chains in large multiple beams arrays}

2 categories of recent missions geostationary satellite require a large number of beams with high directivity:

- fast internet access from/to anywhere, especially in the zones not covered by wired ADSL

- broadcasting high-definition TV programs dedicated to specific linguistic zones, numerous within Europe

Well suited transmit antennas are Active Arrays (operating in $\mathrm{Ku}-$ band around $12 \mathrm{GHz}$ or $\mathrm{K}$-band around $20 \mathrm{GHz}$ ), thanks to following advantages:

- the losses of rather complex beam-formers are "hidden" by high SSPA's gain in the front-end

- the power to be radiated in the various beams is spread over numerous SSPA's, and may be "exchanged" among the various beams to match with instantaneous traffic requirements and mitigate rain attenuation

- "graceful degradation" allows to avoid amplifiers redundancy, for satellites which should operate in a 
harsh environment for 15 to 18 years without repairing.

As a counterpart:

- $\quad$ high directivity needs a large antenna diameter (1 to $2 \mathrm{~m}$ for 0.6 to $1^{\circ}$ beams; depending on $\mathrm{Ku}$ or $\mathrm{K}$ band)

- covering a significant Earth zone means a $6^{\circ}$ to $8^{\circ}$ angular domain as seen from the satellite;

- the Array grating lobes must be rejected out of all terrestrial regions, to comply with frequency spectrum re-use among several systems

The latter conditions require several hundreds of radiating elements and associated active chains, when using classical designs with periodic regular array lattice. This number much exceeds that of phase-control points required to shape and point the antenna pattern(s). Furthermore, this implies a complex Beam Forming Network (analogue or digital). The cost of such an antenna is prohibitive, even if taking advantage of the low power per element for using SSPAs ${ }^{1}$ instead of TWTAs ${ }^{2}$.

Besides, the DC consumption of the transmit section is very high for active DRAs, as the DC-to-RF efficiency of SSPAs is lower than that of TWTAs. This drawback is enhanced if amplitude taper over the array compels to make SSPAs operate at numerous different points from one to others.

Previous Alcatel Alenia Space (become Thales Alenia Space in April 2007) has investigated from several years solutions to mitigate this drawbacks by:

a) drastically reducing the number of parallel RF paths in such large multibeam Active Arrays

b) providing the low required side-lobe level via "spatial tapering" while all SSPAs provide the same RF power.

For comparing various reduction methods and associated algorithms, we begun by the most demanding mission: the 'Ka-band multimedia' with 44 to 64 beams (depending whether we choose as test-case a "pure-European coverage", or one extended to North Africa and Middle East).

\section{Part I: 'Random searching' algorithms for thinned or non-regular arraying}

\section{I-1 Specifications \& initial regular array}

The DRA to be optimised should cover Europe extended to North-Africa and near Middle-East by 64 beams with $0.8^{\circ}$ diameter, and a $42 \mathrm{dBi}$ minimal directivity at $20 \mathrm{GHz}$. This coverage has been the topic of several studies led for ESA \& $\mathrm{CNES}$, and this part sums-up results from [1].

\footnotetext{
1 SSPA: Solid State Power Amplifier

2 TWTA/ Travelling-Wave Tube Amplifier
}

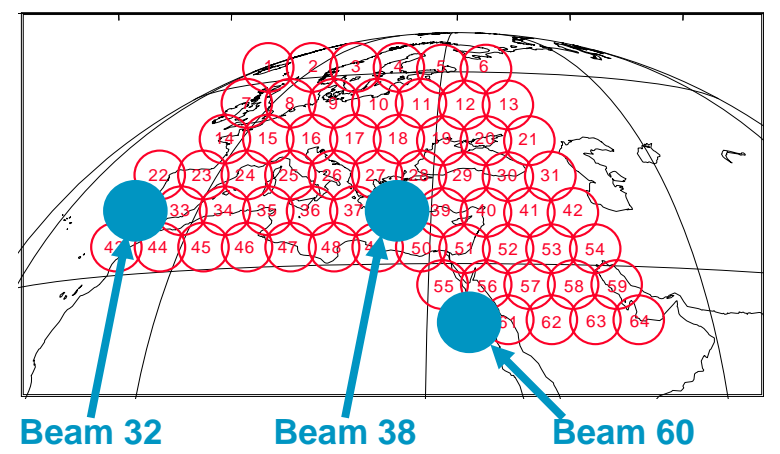

\section{Figure 1: 'Extended Europe' coverag, and 3 chosen beams for focusing optimisations}

The reference antenna used as a starting point for checking various ways to reduce the number of active chains comprises 511 radiating elements arranged according to a regular lattice of equilateral trianbles. The antenna diameter is $1.35 \mathrm{~m}$, and the array spacing $3.8 \lambda$ centre-to-centre. It complies with gain specification on all beams, and rejects grating lobes outside of the Earth.

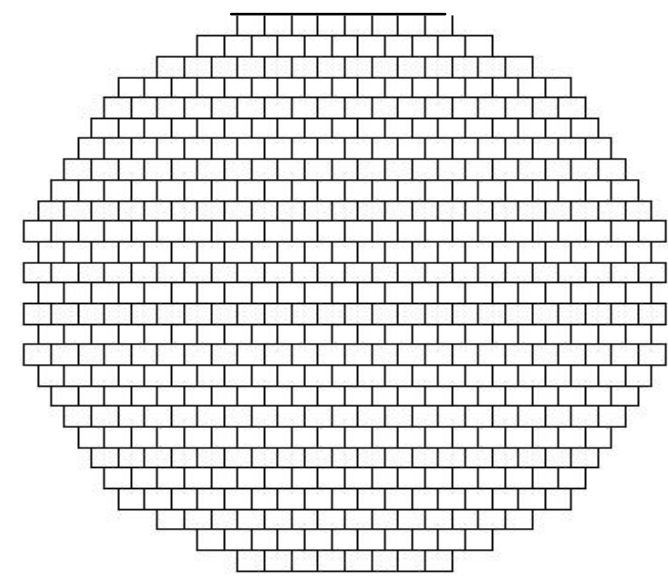

Figure 2: Initial regular array (511 elements)

The cost function to be optimised includes:

- $\quad$ the minimum directivity on all beams (with a strong weight)

- $\quad$ the side-loges level to be minimised, with computing it on 2 different zones: the coverage where it may interfere with other beams using the same sub-band (the frequency re-use scheme is the same as for coverage B: see Figure 4 ); and the rest of the Earth where regulation restricts the level not to disturb other systems.

\section{I-2 Various tested algorithms for array thinning \& non-regular subarrays optimisation}


In the frame of a trainee work within ex-Alcatel Space, we chose 'global optimisation methods' based on 'pseudorandom searching', though they are known as computation time consuming, for following reasons:

a) both array thinning and subarray division deal with discrete variables (for example presence or absence of radiating element in the lattice), thus lead to non continuous nor derivable cost function, badly suited to most analytical 'gradient-type' optimisation tools.

b) due to the very high number of unknowns, the probability for local optimisers to stay trapped in local minima appears high.

c) genetic algorithms and simulated annealing seem well fitted to take into account heterogeneous data in the cost function. Instead of dealing with a highly constrained optimisation - minimizing the number of radiating elements with given sidelobe level for example- it is rather simple to manage the trade off between the number of phase-control points and maximum sidelobe level by tuning the weight of these constraints in the 'fitness' (or 'cost') function, e.g.:

$$
C_{\text {total }}=C_{\text {Initial }}+\alpha \cdot C_{\text {Directiyit }}+\beta \cdot C_{\text {Interfener }}+\gamma \cdot C_{\text {WholEarth }}+\eta \cdot C_{\text {remaininge. }}
$$

\subsection{Genetic algorithms}

They are derived from the way that species evaluate: a set of parameters representing a potential solution is called a chromosome. Instead of working on a single candidate solution at each iteration, a set of possible solutions is considered, called a population.

At each iteration some solutions are randomly selected among the current population, based on their fitness. Better solutions are more likely to be selected, but diversity is guaranteed as all members of a population have some chances to be used to generate new candidates. A new set of solution is fathered from the selected ones, by using process such as mutation and cross-over, generating the population used in next iteration.

The algorithm stops when the solution is sufficiently close to the optimum, or when a predetermined number of iterations is reached.

\subsection{Simulated annealing}

In a metallurgy process,. a material is heated, then slowly cooled in order that each atom finds its minimal energy, leading to a stable state.

To simulate that process, at each iteration, the current solution is replaced by a neighbouring one. The new solution is rated using the cost function. If it is better than the previous one, the transition towards this new state is accepted. If the cost function is degraded, the transition can however be accepted, with a certain probability that decreases when iterating. First iterations then allow exploring the space of all possible solutions, while last iterations will tend to improve a solution already not far from the optimum.

The stop criterion is as mentioned for genetic algorithms.

\subsection{Simulation results for the reference regular array}

Optimisation has been focused on 3 beams among the 64 , as shown on the previous Figure 1:

\section{- $\quad n^{\circ} 38$ near the centre of the coverage}

- $\quad n^{\circ} 32 \& n^{\circ} 60$ at edges of the coverage

First have been computed the performances got with the reference array fed with uniform amplitude, and linear phaseslope for pointing successively towards the centre of each beam.

\begin{tabular}{|c|c|c|c|}
\hline Beam & $\begin{array}{c}\text { Directivity } \\
(\mathrm{dBi})\end{array}$ & $\begin{array}{c}\mathrm{SLL}^{3} \text { over } \\
\text { coverage } \\
(\mathrm{dB} / \mathrm{Max})\end{array}$ & $\begin{array}{c}\text { SLL over Earth, } \\
\text { out of coverage } \\
(\mathrm{dB} / \mathrm{Max})\end{array}$ \\
\hline 38 & 43.7 & -17.5 & -33.7 \\
\hline 32 & 42.4 & -16.3 & -25.6 \\
\hline 60 & 43.5 & -16.9 & -23.9 \\
\hline
\end{tabular}

\subsection{Simulation results for thinned arrays}

Here are an example of one reached solution (beam 38, simulated annealing), and a table summing-up all results:

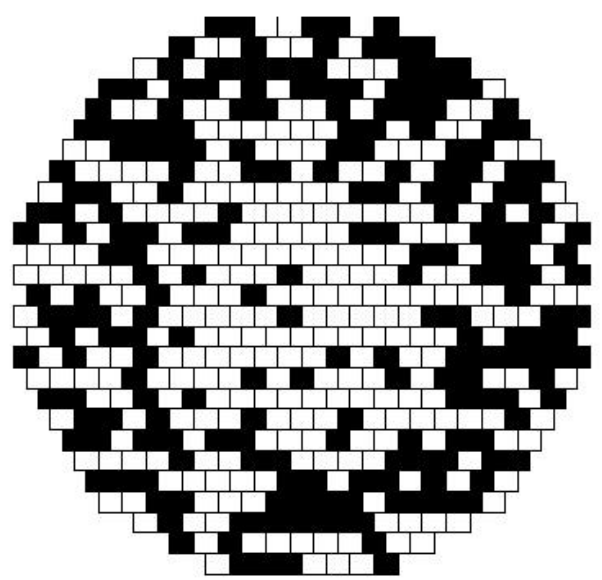

\begin{tabular}{|c|c|c|c|c|c|}
\hline & $\begin{array}{c}\text { Spot } \\
\mathrm{n}^{\circ}\end{array}$ & $\begin{array}{c}\text { Directivity } \\
(\mathrm{dBi})\end{array}$ & $\begin{array}{c}\text { SLL on } \\
\text { coverage }\end{array}$ & $\begin{array}{c}\text { SLL on } \\
\text { Earth }\end{array}$ & $\begin{array}{c}\text { RF-paths } \\
\text { decrease }\end{array}$ \\
\hline \multirow{2}{*}{$\underline{\text { Glgenetic }}$} & 38 & 42.68 & -22.5 & -24.41 & $26 \%$ \\
\cline { 2 - 6 } & 32 & 41.99 & -19.3 & -23 & $19 \%$ \\
\cline { 2 - 6 } & 60 & 42.42 & -21.28 & -22.83 & $25 \%$ \\
\hline \multirow{2}{*}{$\underline{\text { Simulated }}$} & 38 & 42.08 & -24.21 & -22.63 & $42 \%$ \\
\cline { 2 - 6 } & 32 & 42.02 & -20.72 & -24.17 & $28 \%$ \\
\cline { 2 - 6 } & 60 & 42.02 & -22.82 & -24.79 & $42 \%$ \\
\hline
\end{tabular}

As a conclusion, saving from 20 to $40 \%$ is achieved, larger with simulated annealing. We also found that the latter is easoier to tune; so it has been the only one applied to nonregular subarraying

\footnotetext{
${ }^{3}$ SLL: side-lobes level
} 


\subsection{Simulation results for non-regular sub-arraying}

Using simulated annealing method leads to following results:

\begin{tabular}{|c|c|c|c|c|}
\hline $\begin{array}{c}\text { Spot } \\
\mathrm{n}^{\circ}\end{array}$ & $\begin{array}{c}\text { Directivity } \\
(\mathrm{dBi})\end{array}$ & $\begin{array}{c}\text { SLL on } \\
\text { coverage }\end{array}$ & $\begin{array}{c}\text { SLL on } \\
\text { Earth }\end{array}$ & $\begin{array}{c}\text { RF-paths } \\
\text { decrease }\end{array}$ \\
\hline 38 & 42.0 & -25.3 & -26.4 & $53 \%$ \\
\hline 32 & 42.0 & -19.1 & -24.4 & $41 \%$ \\
\hline 60 & 42.0 & -23.8 & -27.3 & $45 \%$ \\
\hline
\end{tabular}

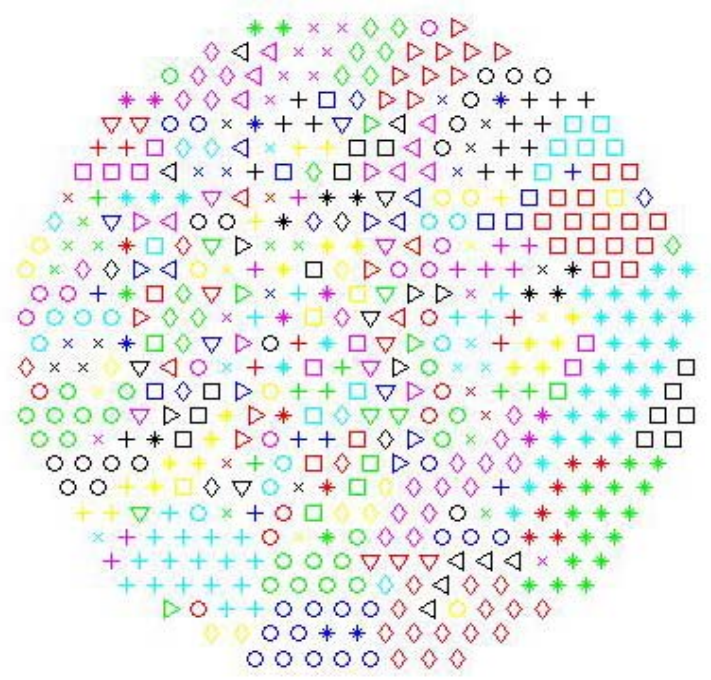

Figure 3: Example of non-regular subarraying solution, reached for beam $n^{\circ} 38$ : all adjacent elements marked with the same symbol can be gathered, that saves half of the active chains versus the reference array

\subsection{Conclusion from results of "random searching" methods}

Both thinning and non-regular sub-arraying allow significant decrease of the number of active chains, with "space taper" as extra-advantage (all feeds or subarrays are connected to amplifiers delivering the same power feeding all sub). Comparisons are in favour of non-regular sub-arraying, optimised by simulated annealing.

However, the following restrictions should be pointed out:

- geometries have been optimised separately for each beam. At least in the limited time of a trainee period, "how to reach solutions compliant for all beams (adding only different phase-slopes) ?" could not be assessed. Trying a global "all beams optimisation" appear to involve a much too high number of criteria to combine (in a single cost function ?).

- No industrial constraint have been taken into account for the non-regular subarraying option, i.e. some restriction on the number of different subarray contours, and if possible simple shapes such as square, rectangle (possibly 'L' or ' $T$ ' types).
To overcome these 2 categories of restrictions, we decide to investigate original analytic methods, able to avoid the "local minima trapping" problem, in collaboration with the "Applied Mathematics lab in Toulouse Academy, led by Pr Masmoudi.

\section{Part II: Analytic algorithms for non-regular arraying}

\section{II-1 Generic test- case, requirements and initial regular array solution}

A "generic" multi-beam coverage (called 'B') has been chosen, slightly different from the ' $\mathrm{A}$ ' one in part I:

- $\quad$ same $0.8^{\circ}$ spot size, same 4-subband frequency reuse in the same band.

- $\quad$ Buy some reduced extension (44 spot-beams instead of 64), and included in a quasi-elliptical contour, to be more generic:

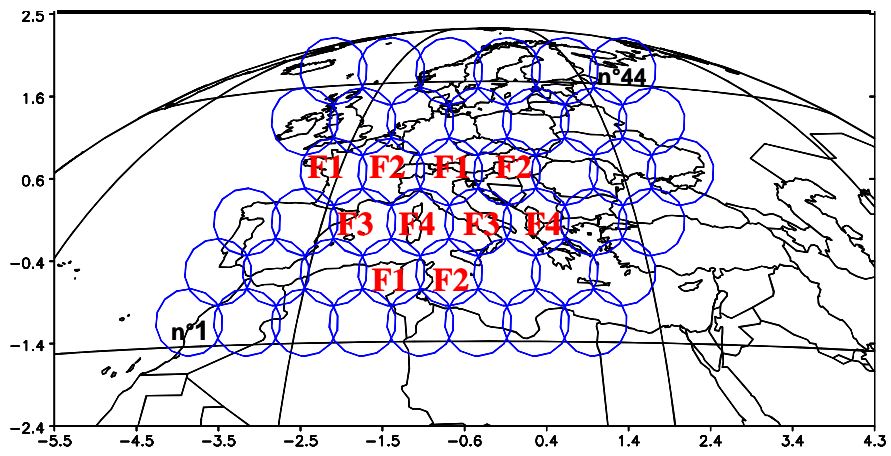

Figure 4: Europe-only coverage by 44 beams with $0.8^{\circ}$ diameter (called ' $B$ ')

The main requirements concerning antenna beams are illustrated (also in a generic way) in the following figure, and associated figures for the text-case are given in the table:

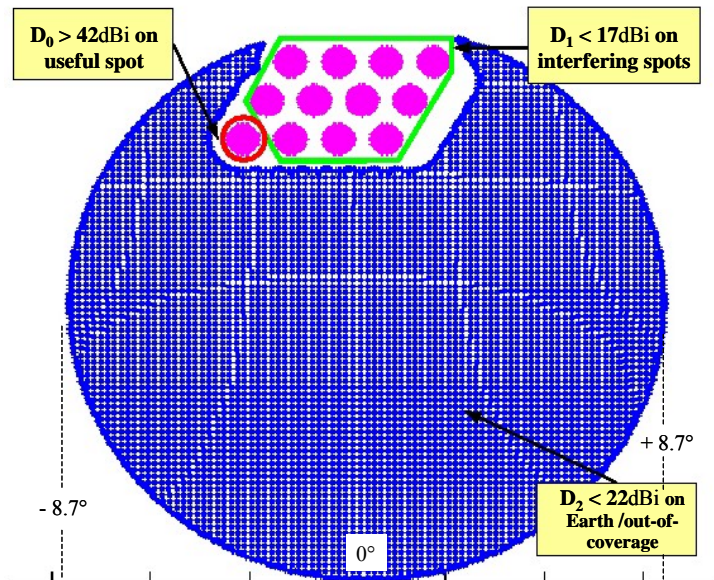

Figure 5: 3 requirements concerning the radiating patterns: the INT(erfering) zone is restricted to the pink spots, using the same subband, and not to their envelope contour drawn in green 


\begin{tabular}{|c|c|}
\hline Direc $^{\text {tivity }}$ over the useful spot " $\operatorname{eoc}^{4 "}{ }^{4} \mathrm{D}_{0}$ & $>42 \mathrm{dBi}={ }^{\prime} \mathrm{UTI} '$ \\
\hline Direc $^{\text {tivity }}$ over the interfering spots $D_{1}$ & $<17 \mathrm{dBi}=$ 'INT' \\
\hline Direc $^{\text {tivity }}$ over Earth, out of the coverag & $\mathrm{i}={ }^{\prime} \mathrm{ISO}$ ' \\
\hline
\end{tabular}

Table A: Quantitative requirements for the chosen test-case

Notice that to ease the optimisation, we don't specify the " $\mathrm{C} / \mathrm{I}$ ", which is the final requirement in terms of "nominal signal C to interferers $(\mathrm{I}=\Sigma \mathrm{i})$ ratio".

Indeed, the C/I computation is quite complicate (and different from one program to another). From our experience, complying with the $\quad i / C<-25 d B \quad$ worstcase requirement for 'INT' warrants an overall $I / C<-15 d B$, whatever is the formula retained to compute the latter, after adding a $\pm 0.05^{\circ}$ antenna pointing error (reached only with a closed-loop antenna self-pointing system towards beacon(s) on ground.

\section{Initial regular array antenna}

It is composed of square radiating elements (abbreviated further as "R.E's") with $(3.2 \lambda)^{2}$ area in the radiating plane. Within a $1.3 \mathrm{~m}$ diameter known from experience as well-fitted to comply with the requirements, there are 529 R.E.

The array lattice is square to allow easy gathering of R.E's. in groups (alias 'subarrays'), keeping for them a rectangular shape compliant with industrial constraints for manufacturing: either it could be short horns, or patch-type subarrays (in the latter case drawn here-after, the basic element is a $(4 \times 4$.) subarray with a classical $0.8 \lambda$ spacing between individual patches.

In a similar way, we deliberately had in mind to keep at most 5 different types of element groups.

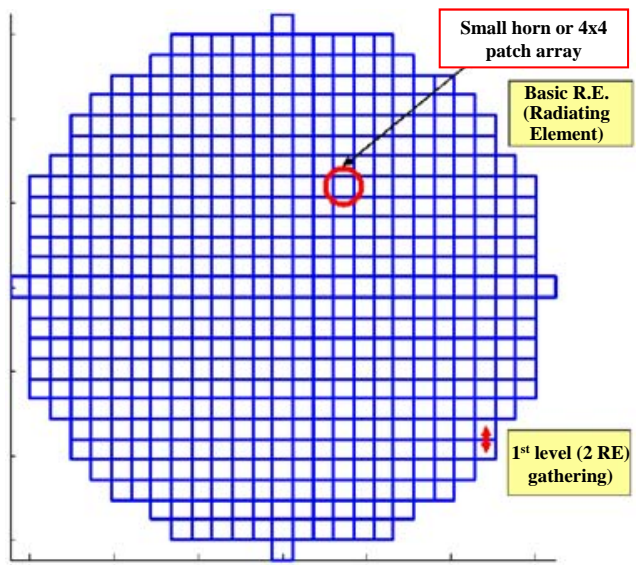

Figure 6: Initial array (529 elements in a regular lattice)

The $(d=3.2 \lambda)^{2}$ array lattice allows a generic design in the following sense:

\footnotetext{
${ }^{4}$ Eoc: edge-of-coverage (more precisely: minimum over the coverage)
}

- As $\quad \sin ^{-1}(\lambda / d)=18.2^{\circ}$

wherever is the pointing of the antenna boresight on the Earth, there will be no "underlaying" grating lobe falling within the Earth disk (which diameter is $17.4^{\circ}$ ), even if taking into account a half-width of the main lobe at its basis:

- "underlaying" grating lobes ? because we start with a regular lattice; so even if a pseudo-random gathering may spread any other nearer grating lobe (which could come from a periodicity with a larger spacing), the grating lobes coming from the basic periodic lattice is still present;

- $\quad$ as the concerned multi-beam coverages used in Kasystems are of limited extension (mostly a continent, here Europe), the "beam scan" (if a beam is assumed to be put successively at the place of each fixed beam) will be much less than the full Earth. So this basic $3.2 \lambda$ spacing lets some margin for further gathering R.E. in larger groups.

The next $\S$ II-2 presents the basics of the analytical optimisation method; then $\S$ II-3 will describe the main results obtained on the test case.

\section{II-2 Non- regular Array option: basics of the optimisation method}

\subsection{Choosing the best parameters and cost-function}

The antenna is made of $n$ feeds (each connected to an active path including a specific phase-control for each beam), and we denote by $a_{\mathrm{i}}$ the complex excitation of feed $i$. The several constraints on the array antenna 'complex excitations' vector $\boldsymbol{a}=\left(a_{j}\right)_{i=1 . . \mathrm{n}}$ and the radiated power over the various zones defined in Figure 5 are

- $\left|a_{i}\right|^{2}=1 / n \quad$ for all radiating elements, as all feeds shall radiate the same power (we normalise the total antenna power is equal to 1$)$;

- $|E(\boldsymbol{a}, x)|^{2} \geq g_{0} \quad$ for all points $x$ in the covered spot, in order to comply with the minimal directivity requirement $D_{0}>U T I$

- $|E(\boldsymbol{a}, x)|^{2} \leq g_{l}$ for all points $x$ in the other spots, in order to minimize interferences in the same sub-band $\left(D_{1}<U T I\right.$ requirement)

- $|E(\boldsymbol{a}, x)|^{2} \leq g_{2}$ for all points $x$ in the isolation zone, i.e. on the Earth, outside the UTI \& INT zones ( $D_{2}<I S O$ requirement).

As the final goal is to reduce the number of antenna feeds by gathering some radiating elements, the idea is to relax the first constraint on the excitation power, and to gather feeds with small excitation modulus. The non-convex first constraint is then replaced by a convex constraint:

$$
\left|a_{i}\right|^{2} \leq 1 / n \quad \text { for all radiating elements. }
$$

The radiated power $|E(\boldsymbol{a}, x)|^{2}$ is a (quadratic) convex function of the excitation vector $\boldsymbol{a}=\left(a_{i}\right)$, and all the constraints are now convex, except the second one. So we replace this inequality by an equality constraint (which is convex); 
instead of maximizing the energy inside the spot coverage, the electrical field should be equal to a given value at the centre of the spot:

- $E\left(\boldsymbol{a}, x_{0}\right)=g_{0}+\delta_{0}$, where $x_{0}$ is the centre of the desired spot, and $\delta_{0}$ a parameter to be tuned.

This will ensure that the radiated power is greater than $g_{0}$ if $\delta_{0}$ is large enough. This works because the solid angle of the spot is small.

Normally the solution a to the original problem is defined up to a constant phase change. Thanks to the last modification, the phase is fixed by imposing $g_{0}+\delta_{0}$ to be a real number (i.e. 'reference phase $0^{\circ}$ at the spot centre'). By reducing the set of possible solutions, we improve the quality of our optimisation problem.

Notice that we don't use decibels for aperture field and radiating one evaluations. It destroys the so important quadratic property of the power, appearing in most constraining equations .

All the constraints are convex: we are sure to reach an optimal solution using a descent method, without being trapped in a local minimum.

In order to find an excitation vector satisfying all these constraints, we define a vector function $\mathbf{F}$, in which each component is related to the compliance with one of these constraints (the component is null if and only if the constraint is satisfied). We finally define the following cost function

$$
\underline{J(\boldsymbol{a})=1 / 2 \|(\boldsymbol{F}(\boldsymbol{a}))^{ \pm} \amalg^{2} .}
$$

where $\mathrm{X}^{+}$means 'positive part of a real number $\mathrm{X}^{\text {'. }}$

The excitation vector satisfies all constraints if and only if $J(\boldsymbol{a})=0$. The idea is then to minimize the functional $J$ with respect to the real and imaginary parts of a, and not to the modulus and phase for linearity purpose. This unconstrained minimization is performed with a Levenberg-Marquardt algorithm $[1,3]$, and provides an optimal excitation vector a for each spot.

\subsection{Data reduction technique: the Singular Value Decomposition (SVD)}

We have seen in the previous paragraph how to find an optimal excitation vector a for each spot on the Earth. If we denote by $s$ the number of spots, we can define a $n \times s$ matrix $\boldsymbol{A}$, in which the $k^{\text {th }}$ column $(1 \leq k \leq s)$ contains the modulus of the optimal excitation vector a corresponding to spot $k$, provided by the previous minimization with the convex inequality constraint on the modulus of $\mathbf{a}$.

In order to easily extract the maximum of information from all these excitations, we will apply the Singular Value Decomposition (SVD) method to this matrix $[2,4]$. The main result of this theory states that the matrix $\mathbf{A}$ has a factorization of the form

$$
\boldsymbol{A}=\boldsymbol{U} \Sigma \boldsymbol{V}^{*}
$$

where $\boldsymbol{U}$ is a $n \times n$ unitary matrix (i.e. $\boldsymbol{U} \boldsymbol{U}^{*}=\boldsymbol{U}^{*} \boldsymbol{U}=\boldsymbol{I}$, identity matrix), $\boldsymbol{V}$ is a $S^{\times} S$ unitary matrix, $\mathbf{V}^{*}$ is the conjugate transpose of $\boldsymbol{V}$, and $\boldsymbol{\Sigma}$ is a $n \times S$ real matrix with non-negative numbers on the diagonal and zeros off the diagonal. The $\Sigma$ matrix contains the singular values $\left(\sigma_{i}\right)$ of $\boldsymbol{A}$, which can be thought of as scalar "gain controls" by which each corresponding input is multiplied to give a corresponding output. A common convention is to arrange the singular values in decreasing order. In this case, the diagonal matrix $\Sigma$ is uniquely determined.

Some practical applications need to solve the problem of approximating a matrix $\boldsymbol{A}$ with another matrix $\tilde{\boldsymbol{A}}$ which has a smaller rank $r$. In the case where this is based on minimizing the Frobenius norm of the difference between $\boldsymbol{A}$ and $\tilde{\boldsymbol{A}}$ under the constraint that $\operatorname{rank}(\tilde{\mathbf{A}})=r$, it turns out that the solution is given by the SVD of $\boldsymbol{A}$, namely

$$
\tilde{A}=\boldsymbol{U} \Sigma \boldsymbol{V} \boldsymbol{V}^{*}
$$

where $\Sigma$ is the same matrix as $\Sigma$ except that it contains only the $r$ largest singular values (the other singular values are replaced by zero). The Frobenius square norm of the difference between $\boldsymbol{A}$ and $\tilde{\boldsymbol{A}}$ is the sum of the square singular values that have not been kept:

$$
\sigma_{r+1}^{2}+\sigma_{r+2}^{2}+\ldots+\sigma_{s}^{2}
$$

and decreases when $r$ is increased $[2,4]$.

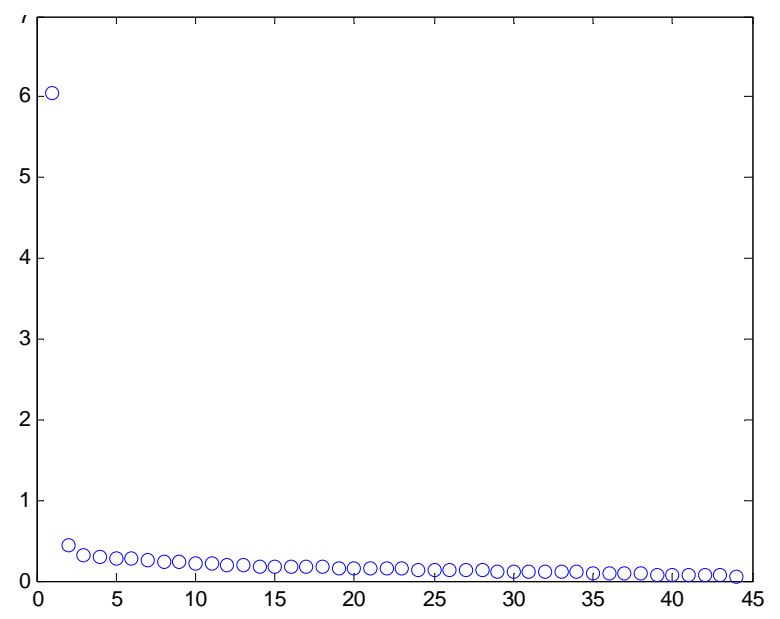

Figure 7: Singular values of optimal excitaions matrix (obtained for our test-case)

Usually, in most applications (as shown on Figure 7, for the present text-case described in $\S \mathbf{I I}-1)$, the decrease of the singular values is such that the greatest one $\sigma_{l}$ is much larger than the others, and they quickly decrease to 0 . If one only keeps the first singular value (i.e. the rank of $\tilde{A}$ is $r=1$ ), the approximation is still very accurate and becomes

$$
\tilde{A}=\sigma_{l} \boldsymbol{u}_{l} \boldsymbol{v}_{l} *
$$

where $\boldsymbol{u}_{l}$ is the first column of $\boldsymbol{U}$ and $\boldsymbol{v}_{l} *$ is the first row of $V^{*}$. Then, the most part of the information contained in $\boldsymbol{A}$ can be expressed with only $\boldsymbol{u}_{1}$. In other words, all modulus of the $s$ excitation vectors are proportional to the vector $\boldsymbol{u}_{1}$. We have finally reduced $s$ excitation vectors to only one. 


\subsection{Topological optimization of the antenna}

Some neighbouring feeds will be merged in order to minimize the number of antenna feeds and hence of phase control points. In such case, the number $n$ of radiating elements will decrease, and as the total power does not change, the constraints on each feed modulus will change. For example, if we merge 2 feeds, we have the following configuration change:

- before gathering in $n$ feeds, with $\left|a_{i}\right|^{2}=1 / n$.

- after merging: n-1 feeds, with $\left|a_{i}\right|^{2}=1 /(n-1)$ for all feeds, except for the two merged ones: $\left|a_{i}\right|^{2}=0.5 /(n-1)$.

In case some neighbouring feeds have comparable excitation modulus, they will be merged according to several predefined geometries. In the following, we will only consider only merging of 2, 3 or 4 feeds together, according to a rectangular contour (which includes the square cases: $1 \times 1$ or $2 \times 2$ initial R.E. $\left.{ }^{5}\right)$.

For a given antenna with $n$ radiating elements, we denote for each radiating element $i$ by

- $r_{i}, 1 \leq r_{i} \leq 4$ : the number of R.E. possibly merged into the feed to which the concerned R.E. belongs;

- $c_{i}^{n}=1 /\left(r_{i} \times n\right)^{1 / 2}$ the theoretical excitation modulus imposed by the constraint of fixed total power, equally shared among all groups;

- $\underline{m}_{i}$ the $i^{\text {th }}$ component of the first vector $\boldsymbol{u}_{l}$ of the singular value decomposition of the excitation matrix $\boldsymbol{A}$ (see previous subsection).

We can now define a cost function measuring the difference between the theoretical and computed excitation modulus:

$$
\underline{I_{k}}=\sum_{\underline{i=1 . . n}}\left(c_{\underline{i}}^{\underline{n}}-m_{\underline{i}}\right)^{2} .
$$

where $k$ suffix is the concerned iteration number. Be careful that this 'cost function' is different from the initial one $J$, which led to optimised excitations separately for each spot.

The geometry of the antenna is optimized by minimising $\underline{I}_{k}$ at each $k$ iteration.

- $\quad$ find the radiating element $i_{0}$ maximizing the error $\left(c_{\mathrm{i}}^{n}-m_{i}\right)$

- gather all possible feeds involving this element, according to the predefined geometries, consider these few new geometries and find the one minimizing the error between the theoretical and computed excitation modulus (see equation (6) );

- if this error is smaller than $I_{k}$, then validate the corresponding gathering, and set $k=k+1$; otherwise stop (i.e. the algorithm has converged).

As a final validation, we check then that the optimised array geometry complies with all initial criteria $\left(g_{0}, g_{1}, g_{2}\right)$.

\footnotetext{
${ }^{5}$ R.E.: Radiating Element, as defined in the initial antenna before
} any gathering

\subsection{Optimisation summary, applied to the chosen test-case}

With a more intuitive wording, we sum-up here-under the mathematical procedure described in paragraphs 2.1 to 2.3, and illustrate with intermediate results for the antenna requirements given in $\S$ II-1 as follows:

- $\quad$ in \$2.1, the excitation vector $\mathbf{a}$ is optimised with continuous possible amplitudes (alias $\left|a_{i}\right|$ modula), and separately (with a different solution) for each spot

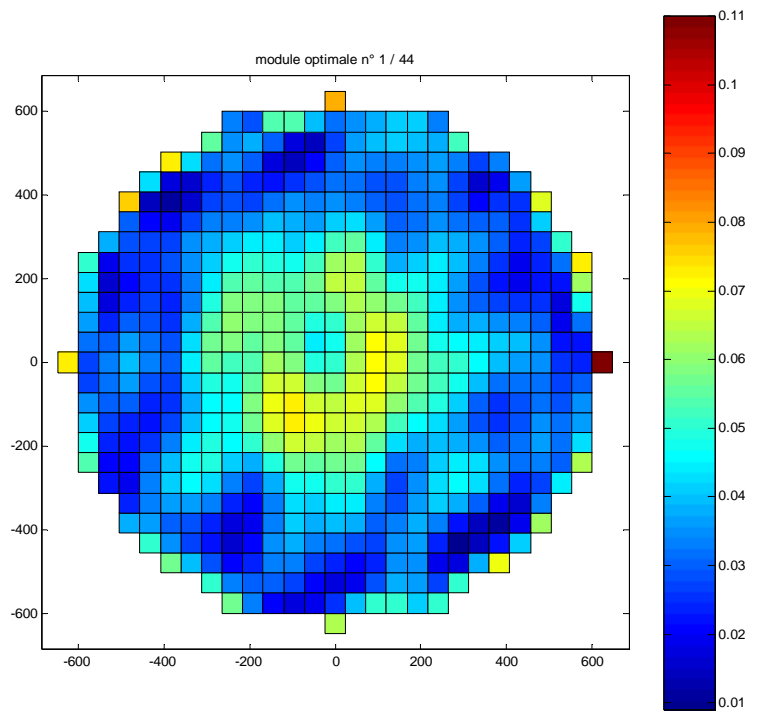

Figure 8: Excitation amplitudes optimised versus $(g 0, g 1, g 2)$ criteria for spot 1 (at bottom-left of the coverage)

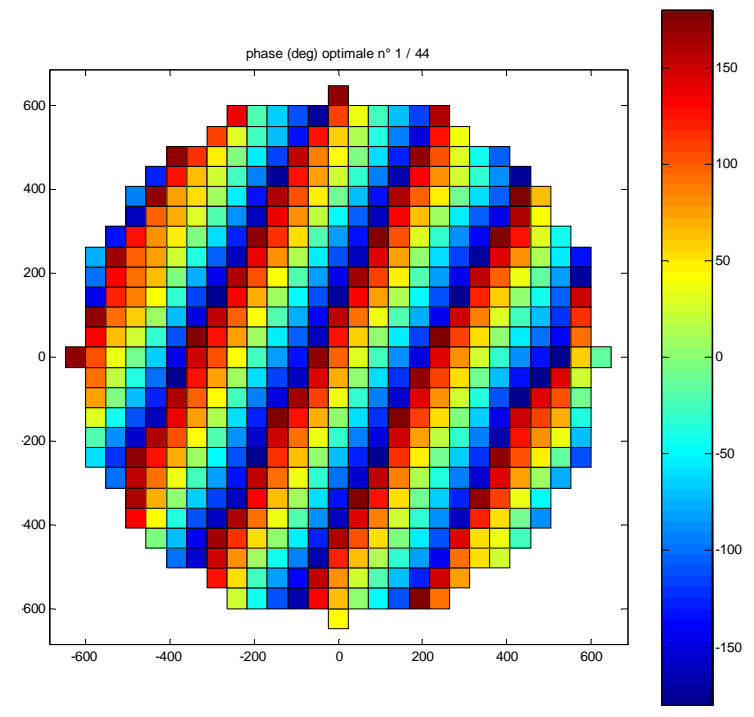

Figure 9 Optimised excitation phases for spot 1

- in \$2.2, the best "average" â (for all spots)was found thanks to the SVD, and keeping only the highest singular value. It is still a vector with 'continuous' excitations modulus 


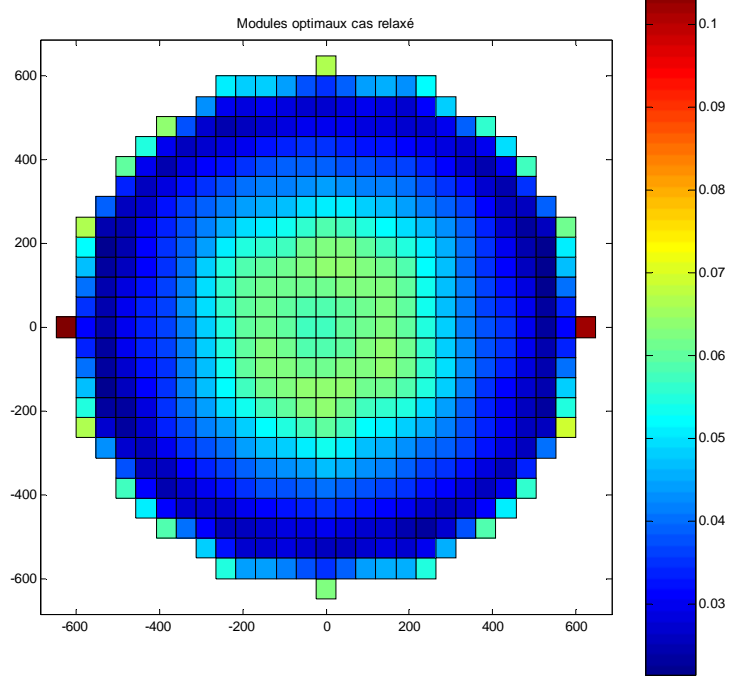

Figure 10: Optimum amplitudes for all spots (from 'SVD')

- $\quad$ in $\S 2.3$, we find the best geometrical gathering to fit the excitation modula with the "quantified" values [ $\left.\left|a_{\mathrm{i}}\right|^{2}=1 /\left(r_{\mathrm{i}}^{6} \times n\right)\right]$ best-approaching Figure 10.

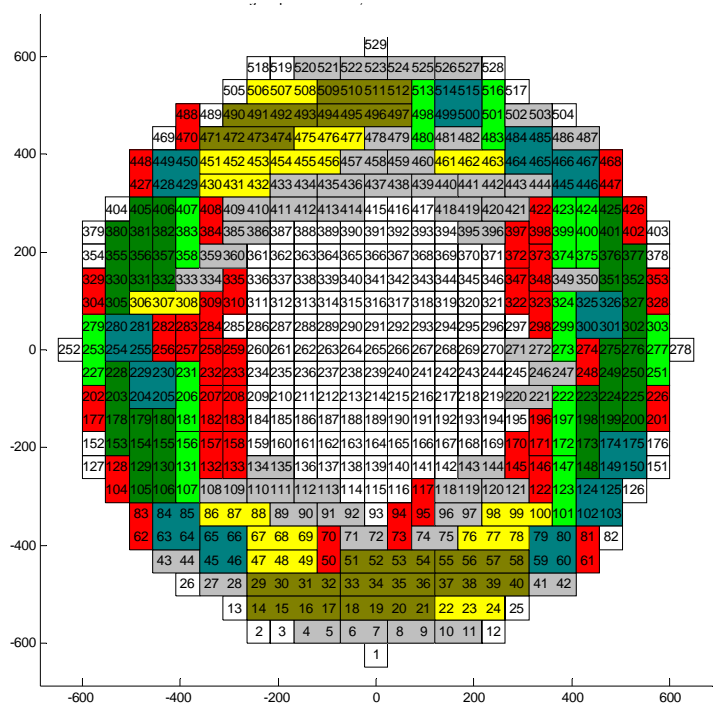

Figure 11: Gathered elements, fed with equal power (1 to 4 elements + only square/rectangular contour)

\section{Non-regular Array option: results obtained for the European multi-beam coverage}

When looking at the antenna geometry issued of the optimisation procedure, as shown in $\$ \mathbf{2 . 4}$, one can see that there is a significant number of elements not gathered at the edges. It's because groups have been created around them, when gathering elements located a little further, nearer to the centre of the array

\footnotetext{
${ }^{6} r_{\mathrm{i}}$ is the group rank, i.e. the number of initial R.E. gathered in it
}

For antenna people, a so high power density increase at the edge is not optimal. So a complementary step is added: reducing the 'single' edge elements ('single' means 'not gathered with others, kept with the minimal R.E. size'):

- starting from the solution found in $\$ 2.4$, the algorithm is then compelled to try all possible gathering including single edge elements.

- $\quad$ this leads to a further decrease of the overall feeds number (from 295 to 283), and to much fewer 'single edge elements'.

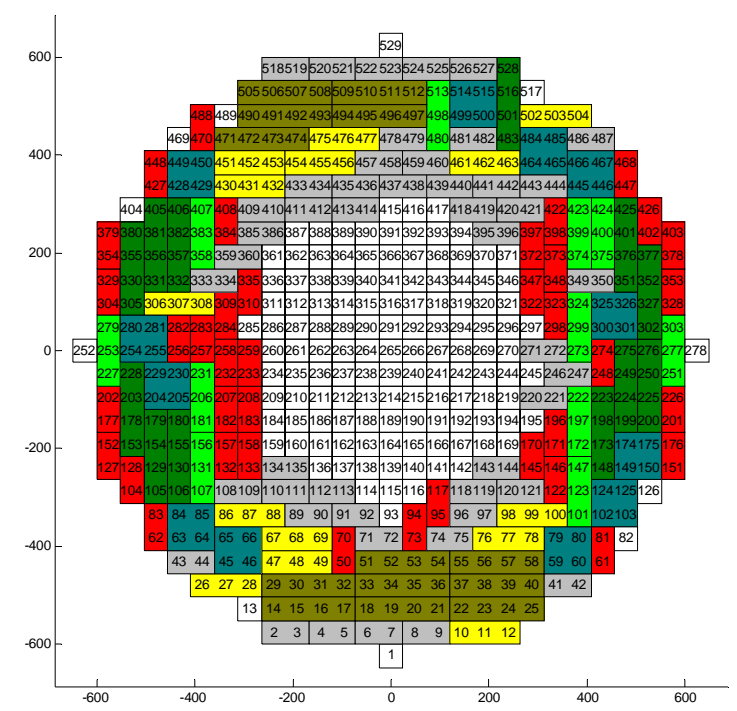

Figure 12: Final non-regular array: 283 feeds instead of 529 initially, thanks to gathering in groups (1 to 4 elements, with square or rectangular contour)

With this solution (array amplitude issued from the optimises groups topology + phases for each spot included in the complex found $a_{i}$ ), we compute all the array performances. They are all compliant with requirements, and even with some margin on the 3 specifications:

- UTI (minimum directivity on the concerned spot: red circle in Figure 13 )

- INT (Max directivity on the interfering spots: blue circles)

- ISO (Max directivity on the Earth, out of the coverage: green circles): 

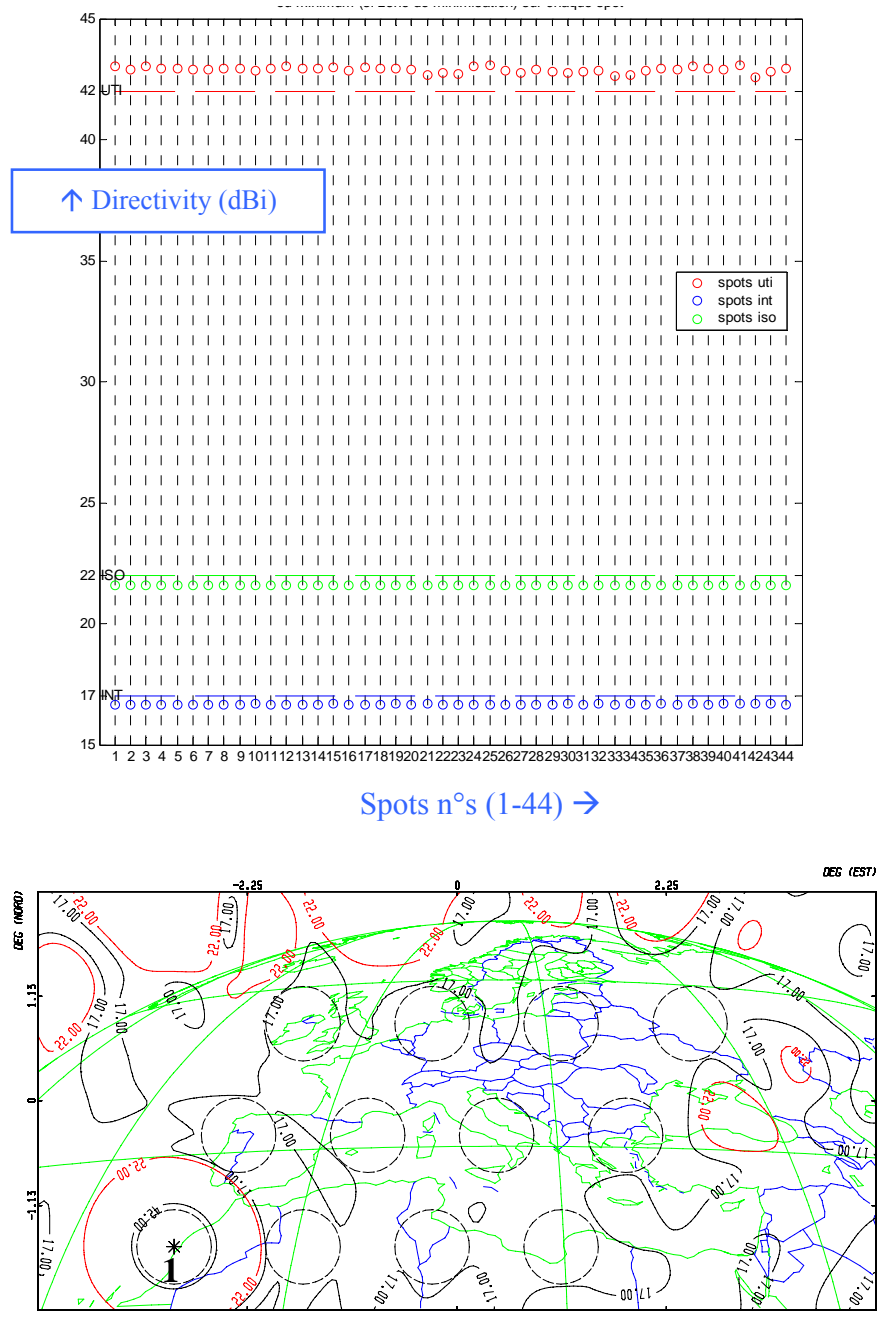

Figure 13: Fully compliant performances of the final solution (upper part); pattern example for spot 1 (lower part)

\section{Conclusion}

We assessed 2 basically different methods, and 3 kinds of optimisation algorithms, for reducing the number of phasecontrol points in large arrays with multiple beams. The main conclusions are:

:- "random searching" type algorithms are well-suited to find good solutions for array thinning, as it deals simply with " 0 or 1 " binary parameters (an element is present or not). Among them, the "simulated annealing" reaches higher active chains reduction, provided some experience for well tuning has been got. From 20 to $30 \%$ elements may be saved without significant performance reduction, and keeping the same overall array diameter; however performances for all beams with a fixed optimised thinning have not been fully computed.

- algorithms based on well-chosen analytic optimisation ("Single Value Decomposition" + "topologic gradient") appeared compulsory to reach safely solutions for elements gathering, with all beams compliant with the chosen typical requirements: up to $\mathbf{5 0 \%}$ number of phase-control have been saved in a non-regular array comprising only 4 kinds of rectangular "subarrays": this induces a low cost increase, compared to the large one coming from decreasing the number of active chains

Both methods present another advantage for industrial implementation: the taper which is compulsory for low sidelobes is provided by the non-regular density of elements feeding points. So in a transmit antenna, all amplifiers would provide the same power, working at the same well-optimised operating point.

\section{References}

[1] C. Guiraud et al: "Reducing DRA complexity by thinning \& splitting into non-regular subarrays, $29^{\text {th }}$ ESA Antenna Workshop (April 2007).

[2] G. H. Golub, C. F. Van Loan: "Matrix Computations", 3rd ed., Johns Hopkins University Press, Baltimore, (1996).

[3] Marquardt. D: “An Algorithm for Least-Squares Estimation of Nonlinear Parameters", SIAM J. Appl. Math., 11, pp. 431-441, (1963). 